\title{
La Universidad de Buenos Aires en la década de 1970: análisis del comunicado de prensa en solidaridad con Rodolfo Puiggrós
}

\author{
The Universidad de Buenos Aires in the 1970s: analysis \\ of the press release expressing solidarity with Rodolfo \\ Puiggrós
}

Recalde, Aritz ${ }^{1}$

${ }^{1}$ Licenciado en Sociología. Magíster en Gobierno y Desarrollo. Director de Posgrado, Universidad Nacional de Lanús,

Argentina.

aritzrecalde@hotmail.com
RESUMEN Este artículo reconstruye el contexto histórico de aparición del comunicado de prensa emitido por las autoridades de la Universidad de Buenos Aires en solidaridad con el historiador y rector de la universidad, Rodolfo Puiggrós, luego de su renuncia. Su gestión intentó ligar los planes científicos y tecnológicos de la institución al nuevo modelo de desarrollo iniciado por Héctor Cámpora y el peronismo en el año 1973. Se hace referencia al proyecto de transformación de la universidad que fue impulsado por Puiggrós y que articuló tradiciones ideológicas marxistas, reformistas y nacionalistas; se introducen algunas claves de lectura para analizar la causa de la renuncia de Puiggrós y se recuperan los argumentos del comunicado de prensa publicado por la Dirección de Prensa y Difusión de la Universidad de Buenos Aires el 5 de octubre de 1973.

PALABRAS CLAVE Historia del Siglo XX; Universidades; Formulación de Políticas; Argentina.

ABSTRACT This article reconstructs the historical context surrounding the emission of a press release by authorities of the Universidad de Buenos Aires, expressing their solidarity with historian and university rector Rodolfo Puiggrós after his resignation. In his term, Puiggrós attempted to fuse the institution's scientific and technological plans with the new development model initiated by Héctor Cámpora and the Peronist movement in 1973. The article makes reference to the project of university transformation promoted by Puiggrós, combining the ideological traditions of Marxism, reformism, and nationalism. A few key points, useful for analyzing the reasons behind Puiggrós's resignation, are presented, and the arguments highlighted within this press release, published by Office of Media and Communications on October 5, 1973, are discussed.

KEY WORDS History, 20th Century; Universities; Policy Making; Argentina. 


\section{INTRODUCCIÓN}

El 5 de octubre del año 1973 la Dirección de Prensa y Difusión de la Universidad de Buenos Aires (UBA) (a), publicó un comunicado (1) en apoyo a la gestión del saliente rector interventor Rodolfo Puiggrós.

La intervención de las universidades se efectuó para adecuarlas al gobierno democrático iniciado en 1973, luego de varios años de implementación de dictaduras y proscripciones políticas. La medida fue anunciada por el ministro de Educación de la Nación, Dr. Jorge Taiana, que leyó el Decreto 35. Entre los considerandos del Decreto se mencionaba:

Que la Liberación Nacional exige poner definitivamente las universidades nacionales al servicio del pueblo, siendo por lo tanto necesaria la reformulación de los objetivos, contenidos y métodos de la enseñanza con la participación de todos los sectores vinculados a la vida universitaria [...] Cámpora decretó la intervención de las universidades nacionales. (2)

Previo a su renuncia, Puiggrós ejerció su cargo de rector de la UBA desde el 29 mayo de 1973, hasta el 17 de septiembre de 1974. Su ingreso y su salida, coincidieron con la asunción y con la posterior renuncia del presidente Héctor Cámpora, de cara al regreso de Juan Domingo Perón a la primera magistratura.

Las causas de la renuncia de Puiggrós fueron confusas. En conferencia de prensa, Puiggrós sostuvo que la solicitud de su renuncia había sido efectuada por el ministro de Educación Jorge Taiana, sin conocimiento de Juan Domingo Perón. Con este último, se comunicó telefónicamente y según Puiggrós, Perón le declaró no conocer la solicitud de dimisión de su cargo. Según fuentes periodísticas, la decisión del pedido de renuncia de Puiggrós fue decidida por Raúl Alberto Lastiri en el ejercicio del Poder Ejecutivo Nacional tras la renuncia de Héctor Cámpora (3).

Al asumir el rectorado, Rodolfo Puiggrós era un reconocido historiador, periodista, docente y militante político. En los años treinta, se afilió al Partido Comunista y, frente al proceso político iniciado en el año 1946, fundó con otros intelectuales y dirigentes el Movimiento Obrero Comunista (MOC). El MOC nació como un desprendimiento del comunismo fruto de la negativa de este partido de otorgar apoyo al programa de gobierno peronista en los años cuarenta.

Tal cual se pudo observar en su gestión a cargo del rectorado, existió un intento de articular las concepciones de la izquierda marxista con postulados nacionalistas en el marco de un apoyo al gobierno de Héctor Cámpora.

\section{ACERCA DE LA INVESTIGACIÓN}

Para poner en contexto el "comunicado de prensa", principal fuente documental de este análisis, se consultaron además los siguientes documentos:

- Resoluciones y boletines informativos publicados por la UBA desde el 29 mayo de 1973 hasta el 19 de septiembre del año 1974.

- Prensa periodística publicada desde el 29 mayo de 1973 hasta el 6 de septiembre del año 1974.

Las fuentes documentales fueron recopiladas por Delia Carnelli de Puiggrós y corresponden a su archivo personal, el cual fue donado a la Secretaría de Derechos Humanos de la Nación (b). El recorte temporal se corresponde al período que se inicia con la fecha de asunción de Rodolfo Puiggrós como rector interventor de la UBA y finaliza con los últimos documentos conservados por su esposa antes del exilio de ambos en México (c).

El comunicado de prensa es un material escasamente difundido por las investigaciones históricas. Los estudios sobre la universidad argentina, se han abocado en pocas ocasiones a investigar el proyecto impulsado en el año 1973 que supuso una nueva forma de organizar la educación superior en su relación con la economía, la sociedad, la política y la cultura del país.

\section{EL COMUNICADO DE PRENSA}

De la lectura de este comunicado (Figura 1) se puede deducir que existió un acuerdo 
Figura 1. Comunicado de prensa emitido por las autoridades de la Universidad de Buenos Aires el 5 de octubre de 1973.

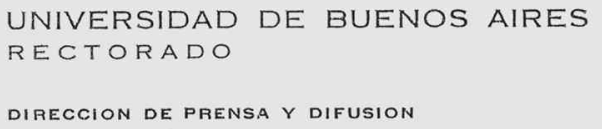

\section{COMUNICADO DE PRENSA}

LOS AQUI PRESENTES, EL SECRETARIO GENERAL DE LA UNIVERSIDAD DE BUENOS AIRES - A CARGO DEL RECTORADO-, Y LOS DELEGADOS INTERVENTORES EN LAS CASAS DE ESTUDIO DEPENDIENTES DE LA MISMA, DESIGNADOS POR EL PROFESOR RODOLFO PUIGGROS, DECLARAMOS:

1) Nuestra total solidaridad con el compañero Puiggros, tanto en cuanto a la politica por él encarada en este proceso de Reconstrucción en nuestro ámbito especifico, como en lo que respecta a su persona, que se ha revelado una vez más, como lo ha hecho sin defección alguna en más de 25 años, como disciplinado soldado de la Causa del Pueblo y de su Movimiento Polltico liderado por el Tte. Gral. Juan Domingo Perón.

11) Nuestra decisión inquebrantable de continuar, los lineamientos de la politica por él inaugurada, en los dlas que restan hasta el 12 de octubre para tratar de implementar las metas que se sintetizan en la aspiración que el estudio, la investigación y el trabajo estén al servicio de nuestra Patria y de su Pueblo, lo que sólo se alcanzará en el clima de orden y disciplina que caracterizó la gestión inaugurada con el Profesor Puiggrós.

Dicha politica concreto, amén del levantamiento de las medidas represivas en el ámbito académico, ideológico y politico, los siguientes logros:

a) Democratización de la enseñanza.

b) Ingreso irrestricto a las Facultades y demás Casas de estudio dependientes de esta. 
c) Cursos regulares para todos los alumnos.

d) Inserción de la Universidad en la Comunidad Nacional.

e) Ruptura con la dependencia cultural.

f) Investigación cientifica al servicio de los intereses nacionales y populares.

111) En los dias que restan, hasta la histórica asunción de la Presidencia de la Nación por parte del Tte. Gral. Perón, por tercera vez, se procederá a implementar las siguientes medidas:

1) Designar al Profesor Rodolfo Puiggros como Profesor Emérito de la Facultad de Filosofía y Letras, a solicitud del conjunto de la Comunidad Universitaria, en acto público a realizarse el próximo miércoles 10 en el Salón de Actos de la facultad de Medicina.

2) De acuerdo con 10 encomendado por el Tte.Gral.Juan Domingo Perón, hacerle entrega del proyecto elaborado por esta Intervención acerca del reordenamiento integral de la Universidad.

3) Entregar al señor ministro de Educación, Dr.Jorge A. Taiana nuestra respuesta referida a los lineamientos generales que deberla contener la futura ley universitaria.

4) Siguiendo la tónica adoptada por los diferentes sectores del Gobierno Nacional, proceder durante la próxima semana a convocar asambleas en cada facultad, en las que se realice un balance de lo actuado por la Intervención.

5) Asegurar todas las condiciones para que el 12 de oc.1 . 
Figura 1. Continuación.

\section{$-3-$}

tubre -fecha en que asume el gobierno el General Perón- la Universidad esté en completo orden, caracteristica que ha signado el gobierno del Compañero Puiggrós. En ese sentido, y de común acuerdo con las agrupaciones estudiantiles y el concurso de los no docentes y docentes se procederá a la limpiezá de las facultades e Institutos, un aporte más a la Reconstrucción Nacional.

\section{ERNESTO VILLANUEVA \\ Secretario General a/c de Rectorado}

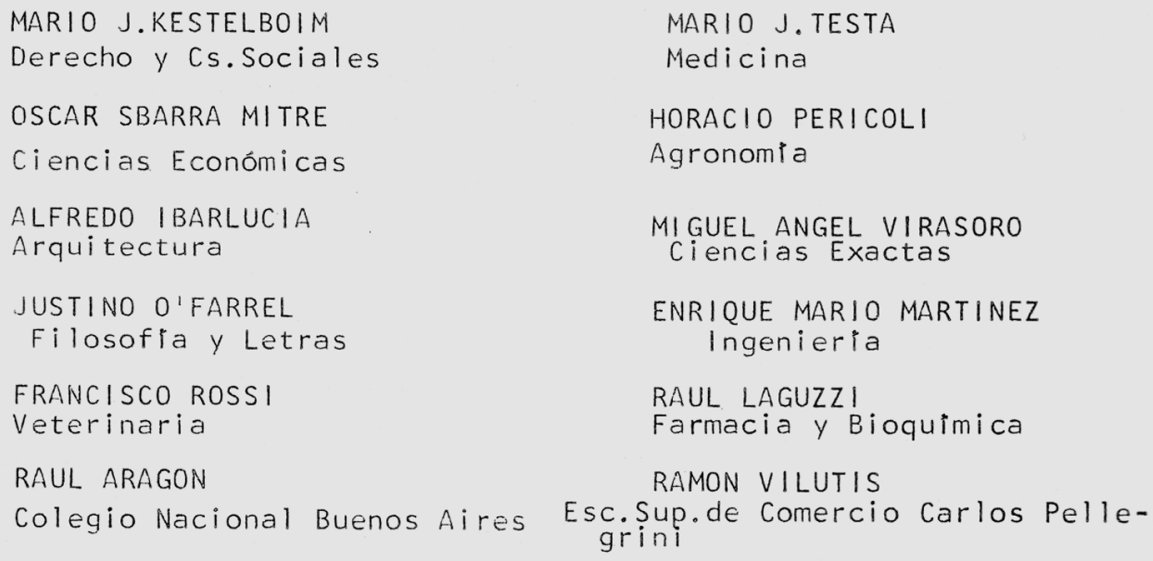

Buenos Aires, 5 de octubre de 1973.- 
generalizado en el respaldo al saliente Rector: firmaron el documento el Secretario General de la institución, diez decanos y los rectores de la Escuela Superior de Comercio Carlos Pellegrini y del Colegio Nacional de Buenos Aires, ambos dependientes de la UBA. No aparece la firma del decano de la Facultad de Odontología, Dr. Alberto F. Banfi, propuesto para el cargo de rector en reemplazo de Puiggrós. La designación de Banfi se efectuó mediante el Decreto $1574 / 73$. Finalmente, Banfi pidió su relevo y asumió el rectorado Ernesto Villanueva (7).

Por otro lado, la salida de Puiggrós fue cuestionada por la Juventud Universitaria Peronista (JUP) que ocupó en forma de protesta, todas las facultades de la UBA (8).

El comunicado se organizó en tres puntos. El punto I se centra en el apoyo a la figura y a la persona del rector saliente. Con esa finalidad, el texto estableció una "total solidaridad con el compañero Puiggrós, tanto en cuanto a la política por él encarnada en este proceso de Reconstrucción de nuestros ámbito específico, como lo es a su persona" (1). Los firmantes del documento resaltaron el compromiso de Puiggrós con el proyecto político peronista.

El punto II hace hincapié en la necesidad de ratificar y de profundizar los cambios institucionales introducidos en la UBA a partir del 29 de mayo. Los firmantes mencionaron la importancia de ratificar los "logros" de la gestión. Entre ellos, rescataron la democratización de la enseñanza, el ingreso irrestricto a la institución, la inserción de la Universidad en la comunidad nacional y la ruptura de lo que denominaron como la dependencia cultural.

La propuesta de insertar la universidad en la comunidad nacional, fue un rasgo característico de la iniciativa institucional, que se preocupó por vincular las acciones académicas con los planes y proyecto de los organismos públicos y no gubernamentales de la región.

Tras su renuncia, Puiggrós presentó un resumen de gestión denominado las “90 medidas más importantes de la Universidad Nacional y Popular de Buenos Aires, en los primeros 90 días de gobierno universitario" (9). En su informe, el rector resaltó la eliminación del aparato represivo, la creación de becas estudiantiles y la promoción de un sistema de salud para los alumnos. La democratización de la enseñanza y el ingreso irrestricto, buscaban derogar las prohibiciones y las regulaciones impuestas a la universidad por los militares que antecedieron la intervención impulsada por el gobierno democrático. En el plano docente, la gestión impulsó la reincorporación de los profesores cesanteados por cuestiones políticas desde el año 1955.

Como mencionamos, los firmantes del comunicado resaltaron la importancia de continuar con la acción tendiente a superar la "dependencia cultural". Con la finalidad de alcanzar dicho objetivo, se promovieron reformas de planes de estudio en gran parte de las facultades de la UBA. Asimismo, se revisaron los vínculos entre la UBA y las empresas multinacionales que podían desviar la producción nacional de conocimiento. Con dicho objetivo, Rodolfo Puiggrós sancionó una Resolución que sostenía "déjase sin efecto el convenio celebrado por Resolución No 1615/66 con la Fundación Ford para la formación de especialistas en Economía Agrícola" (10 p.103).

Entre las medidas contra la "dependencia cultural", cabe destacar, que se designó como presidente de la editorial universitaria EUDEBA al intelectual nacionalista Arturo Jauretche. Al respecto, Puiggrós sostuvo:

\footnotetext{
...vamos a publicar las obras completas del doctor Ramón Carrillo, gloria del movimiento peronista y de la ciencia argentina. Vamos a publicar las obras completas de Leopoldo Lugones. Y vamos a publicar libros de todos los escritores latinoamericanos, tratando de hacer de EUDEBA una editorial continental, con sucursales en todas las capitales del continente latinoamericano y abierta también al tercer mundo. (11)
}

Finalmente, Jauretche publicaría el pensamiento de Héctor Cámpora, de Omar Torrijos, de Velasco Alvarado y de Salvador Allende.

En el comunicado, los firmantes mencionaron la importancia de articular la investigación científica con "los intereses nacionales y populares". Con dicha finalidad, Puiggrós y su reemplazante Ernesto Villanueva promovieron diversas medidas. Entre ellas, se destacan: 
- La creación del Instituto del Tercer Mundo "Manuel Ugarte", que implementó jornadas de debate y promovió investigaciones sobre cuestiones latinoamericanas y del tercer mundo.

- La apertura de los Centros Pilotos de Investigación Aplicada (CEPIA).

En este contexto, resalta la decisión del rector interventor Ernesto Villanueva tomada el 21 de noviembre de 1973 que a través de la Resolución № 895 estableció:

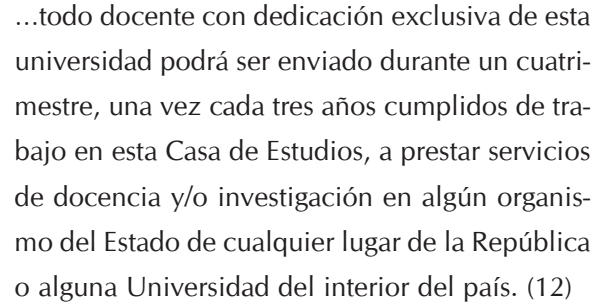

En el punto III del comunicado se realiza una mención especial a la decisión de designar a Puiggrós como Profesor Emérito de la Facultad de Filosofía y Letras. Finalmente, en la Resolución 629/73 fue declarado Profesor Extraordinario Emérito de la UBA.

Cabe destacar que, en este apartado, los firmantes del comunicado mencionaron la entrega al Ministro de Educación de una respuesta a los lineamientos generales para una nueva ley universitaria. Esta última, fue sancionada el 14 de marzo del año 1974 y lleva el número 20.654. En su Art. 1, la nueva "Ley orgánica de las universidades nacionales" estableció que:

Las Universidades Nacionales son comunidades de trabajo que integran el sistema nacional de educación en el nivel superior con el fin de impartir enseñanza, realizar investigación, promover la cultura nacional, producir bienes $y$ prestar servicios con proyección social y, haciendo los aportes necesarios y útiles al proceso de liberación nacional, contribuir a la solución de los grandes problemas argentinos. (13)

El comunicado culminó con la decisión de los firmantes de asegurar las condiciones de institucionalidad para la asunción del gobierno de Juan Domingo Perón el 12 de octubre del año 1973, cuestión que se cumplió.

\section{DISCUSIÓN}

El comunicado permite divisar algunos rasgos de la propuesta universitaria del tercer gobierno peronista. Tal cual se observa, la iniciativa puso en debate muchos aspectos del funcionamiento de la UBA y de las tradiciones académicas liberales y reformistas. En ese contexto, se desenvolvieron algunos intentos de articular la ciencia con el desarrollo nacional independiente, en el marco de una participación activa de la juventud en el contexto político de la época.

Asimismo, el comunicado expresa las fuertes fracturas políticas que se produjeron en el contexto. La confusa salida del rectorado de Puiggrós, fue un Ilamado de atención sobre las dificultades que enfrentó la gestión del gobierno nacional. Finalmente, las iniciativas de modificación de la UBA quedaron inconclusas fruto de una escalada de violencia política que concluyó en la dictadura cívico-militar iniciado en marzo del año 1976.

\section{NOTAS FINALES}

a. La intervención cambió el nombre de la institución que pasó a denominarse Universidad Nacional y Popular de Buenos Aires.

b. El archivo personal de Delia Carnelli de Puiggrós se encuentra actualmente en proceso de digitalización en el Archivo Nacional de la
Memoria, dependiente de la Secretaría de Derechos Humanos de la Nación $(4,5)$.

c. En el exilio en México, Delia Carnelli de Puiggrós realizó una recopilación de recortes periodísticos. Parte de ese archivo, correspondiente al período 1973-1977, se encuentra actualmente digitalizado y puesto a disposición para consulta pública en la página web de la Biblioteca Rodolfo Puiggrós de la Universidad Nacional de Lanús (6). 


\section{REFERENCIAS BIBLIOGRÁFICAS}

1. Universidad de Buenos Aires, Dirección de Prensa y Difusión. Comunicado de prensa. Buenos Aires: UBA; 5 oct 1973. [Material de archivo]. Localizado en: Archivo personal Delia Carnelli de Puiggrós.

2. Confirmóse la designación de Puiggrós: Cámpora decretó la intervención de las universidades nacionales. Diario La Opinión. 29 may 1973. [Material de Archivo] Localizado en: Archivo personal Delia Carnelli de Puiggrós.

3. Puiggrós: "Todo es confuso". Crónica de la Mañana. 4 oct 1973. [Material de archivo]. Localizado en: Archivo personal Delia Carnelli de Puiggrós.

4. Torres Molina R. El archivo de Rodolfo Puiggrós. Tiempo Argentino [Internet] 23 ene 2012 [citado 10 nov 2012]. Disponible en: http://tiempo.infonews.com/notas/archivo-derodolfo-puiggros

5. Enzetti D. El baúl del pensamiento nacional. Tiempo Argentino [Internet] 23 ene 2012 [citado 10 nov 2012]. Disponible en: http://tiempo.infonews.com/notas/baul-del-pensamiento-nacional

6. Universidad Nacional de Lanús, Biblioteca Rodolfo Puiggrós. Archivo Periodístico del exilio argentino en México Delia Carnelli de Puiggrós [Internet] 2002 [citado 10 nov 2012]. Disponible en: http://www.unla.edu.ar/greenstone/cgibin/library.cgi? site $=$ localhost $\& a=p \& p=$ about $\& c$ $=$ archived $\& \mathrm{I}=\mathrm{es} \& \mathrm{w}=\mathrm{utf}-8$
7. Banfi asume funciones. Crónica de la Mañana. 3 oct 1973. [Material de archivo]. Localizado en: Archivo personal Delia Carnelli de Puiggrós.

8. La universidad sin rector: no asumió ayer el Dr. Banfi. Mayoría. 4 oct 1973. [Material de archivo]. Localizado en: Archivo personal Delia Carnelli de Puiggrós.

9. Pruiggrós R. "90 medidas más importantes de la Universidad Nacional y Popular de Buenos Aires, en los primeros 90 días de gobierno universitario". En: La universidad del pueblo. Buenos Aires: Ediciones de Crisis; 1974.

10. Pruiggrós R. La universidad del pueblo. Buenos Aires: Ediciones de Crisis; 1974.

11. Universidad de Buenos Aires, Dirección de Prensa y Difusión. Conferencia de prensa ofrecida por el señor interventor en la Universidad Nacional de Buenos Aires el 12/VI/73. [Material de archivo]. Localizado en: Archivo personal Delia Carnelli de Puiggrós.

12. Universidad de Buenos Aires. Resolución $N^{\circ}$ 895, 21 nov 1973. [Material de archivo]. Localizado en: Archivo personal Delia Carnelli de Puiggrós.

13. Ministerio de Cultura y Educación. Ley $\mathrm{N}^{\circ}$ 20.654. En: Ley Universitaria No 20.654: Serie Educación Legislativa Argentina 5 [Internet]. Buenos Aires: Centro Nacional de Documentación e Información Educativa; 1974. Disponible en: http://www.bnm.me.gov.ar/giga1/documentos/EL0 02644.pdf

\section{FORMA DE CITAR}

Recalde A. La Universidad de Buenos Aires en la década de 1970: análisis del comunicado de prensa en solidaridad con Rodolfo Puiggrós. Salud Colectiva. 2012;8(3):315-322.

Recibido el 1 de agosto de 2012

Versión final presentada el 5 de noviembre de 2012

Aprobado el 23 de noviembre de 2012 